J. Natn. Sci. Coun. Sri Lanka 198311 (1): 41 - 50

\title{
Mathematical Model of Filariasis
}

\author{
K. TENNAKONE
}

Department of Physics, Ruhuna University College, Matara, Sri Lanka

(Date of receipt: 26 August 1982)

(Date of acceptance: 24 March 1983)

\begin{abstract}
A model of man-parasite-mosquito interaction in filariasis is formulated with a system of differential and integrodifferential equations. The model predicts that when the product of population densities of human beings and mosquitoes exceed a critical value, the system becomes unstable and the number of infectives begins to increase exponentially with time approaching an asymptotically stable equilibrium. When this product is less than the critical value, the starting point is asymptotically stable and the number of infectives decreases exponentially with time. It is suggested that because of the varying weather conditions, the disease does not progress very far in the direction of either of these asymptotic equilibria. Data on incidence of filariasis in the southern coastal belt of Sri Lanka is presented to support the model. The implications of the model on eradication of filariasis is discussed.
\end{abstract}

\section{Introduction}

Filariasis is a world health problem. Vast regions of Asia, Latin America, Africa and the Pacific have become a permanent habitat for the disease. Although mosquito control programmes and chemotherapy is reducing its incidence in some areas, the complete eradication seems to be difficult . $4,5,6,11,12$.

The life cycle of the filarial parasite is well known, ${ }^{2,5}$ The definitive host is man, adult worms harboured in the lymphatic system discharge ricrofilariae into the blood stream. The intermediate host is a mosquito, in which the microfilariae taken with a blood meal from the definitive host undergo further development and reach the infective stage in $10-20$ days. When the infective larvae are inoculated into a human being, the sexually matured worms are developed after an incubation period of $6-12$ months. Here the host-parasite interaction is considerably different from that in malaria." Again in filariasis the immunity to the parasite is low and even with drug treatment, the rate of removal of the infectives is small. In this investigation we take these facts into account and construct a mathematica) model of filariasis (mathematicaj nodels of host-parasite systems are discussed). ${ }^{3,7,8}$ The data on incidence of this disease in the southern coastal belt of Sri Lanka is used to test the model.

\section{The Model}

We assume that mosquitoes and human beings are distributed uniformly with population densities $N$ and $n$ respectively. A human susceptible is regarded as infected when his blood contains sufficient microfilariae to infect the mosquitoes. If $N^{*}$ and $n^{*}$ are the population densities of infected human beings and infected mosquitoes, the 
rate at which the latter would interact with the human susceptibles is proportional to $\left(N-N^{*}\right) n^{*}$. Since the onset of the disease occurs a fter a period $\tau$ froin the beginning of exposure to the infected mosquitoes, and the probability of acquiring the infection depends on the integrated intensity of biting by infected mosquitoes during this period. We assume that human infectives are produced at a rate proportional to

$$
\int_{t-\tau}^{t}\left[N(x)-N^{*}(x)\right] n^{*}(x) d x
$$

As the infectives are also removed (due to development of immunity or drug treatment) at a rate proportional to their number density, we write

$$
d N^{*} / d t=b \int_{t-\tau}^{t}\left[N(x)-N^{*}(x)\right] n^{*}(x)-r N^{*}
$$

Where $b$ and $r$ are constants. The rate at which the mosquito susceptibles interact with human infectives is proportional to $\left[n-n^{*}\right] N^{*}$, and since microfilariae undergo metamorphosis within the mosquito, ready to be inoculated into the definitive host rather quickly ( 10 days, small compared to $\tau$ ), for the purpose of the model we ignore this time lag and write the rate equation for development of infected mosquitoes as,

$$
d n^{*} / d t=a\left[n-n^{*}\right] N^{*}-s n *
$$

Where $a$ and $s$ are constants. It should be noted that in the approach we have adopted here, a separate rate equation is not written for the development of parasites within the hosts. In fact the integrodifferential equation (1) arises from this short circuiting. The present approach where the man-parasite-mosquito interaction is included in two steps is more amenable to mathematical analysis.

It is impossible to obtain exact analytical solutions of coupled integrodifferential and differential equations (1) and (2). However, a great deal can be learnt about the form of the solution by studying the system near the critical points. As a first approximation we assume that the total population densities $N$ and $n$ of human beings and mosquitoes remain constant.

There are two critical points corresponding to $d N^{*} / d t=d n^{*} / d t=0$, a node or a saddle point at the origin $N^{*}=n^{*}=0$, and a node $N^{*}=N_{\infty}^{*}, n^{*}=n_{\infty}^{*}$ approached as $t \rightarrow \infty$,
given by the equation;

$$
b\left(N-N_{\infty}^{* *}\right) n_{\infty}^{*} \int_{t-\cdots}^{t} d x=r N_{\infty}^{*}
$$




$$
a\left(n-n_{\infty}^{*}\right) N_{\infty}^{*}=s n_{\infty}^{*}
$$

whose solution is

$$
\begin{aligned}
& N_{\infty}^{*}=(N n-r s / a b \tau)(n+r / b \tau)-1 \\
& n_{\infty}^{*}=(N n-r s / a b \tau)(N+a / s)-1
\end{aligned}
$$

The above critical point is an asymptotically stable node (Appendix). Under present day conditions, the hosts-parasite system will not develop very far in the forward direction of such an asymptotic equilibrium and the behaviour near the critical point $N^{*}=n^{*}=0$ more important. Around this point. when $N^{*}, n^{*}$ are small, neglecting the second order terms we can write (1) and (2) in the form,

$$
\begin{aligned}
& d N^{*} / d t=b N \int_{t-\tau}^{t} n^{*}(x) d x-r N^{*} \\
& d n^{*} / d t=a n N^{*}-s n^{*}
\end{aligned}
$$

Eliminating $N^{*}$ between (7) and (8) we obtain,

$$
d^{2} n * / d t^{2}+(s+r) d n * / d t+(s r) n^{*}=a b n N \int_{t-\tau}^{t} n^{*}(x) d x
$$

The linear equation (9) will have solutions of the form $n^{*}=($ constant $) e^{k t}$ if,

$$
k^{2}+(s+r) k+s r=(a b n N)\left(1-e^{-k \tau} \cdot\right)-1
$$

The characteristic equation (10) has two and only two roots both real (Figure 1)

$$
\begin{aligned}
& k_{1}<0, k_{2}>0 \text { if } n N>s r / a b \tau \\
& k_{1}<0, k_{2}<0 \text { if } n N<s r / a b \tau
\end{aligned}
$$

and the solutions of (7) and (8) can be written in the form,

$$
\begin{aligned}
& n^{*}=A e^{k_{1} t+B e^{\cdot k_{2} t}} \\
& N^{*}=(1 / \text { an })\left(A\left(k_{2}+s\right) e^{k_{1} t}+B\left(k_{2}+s\right) c^{k_{2} t}\right)
\end{aligned}
$$



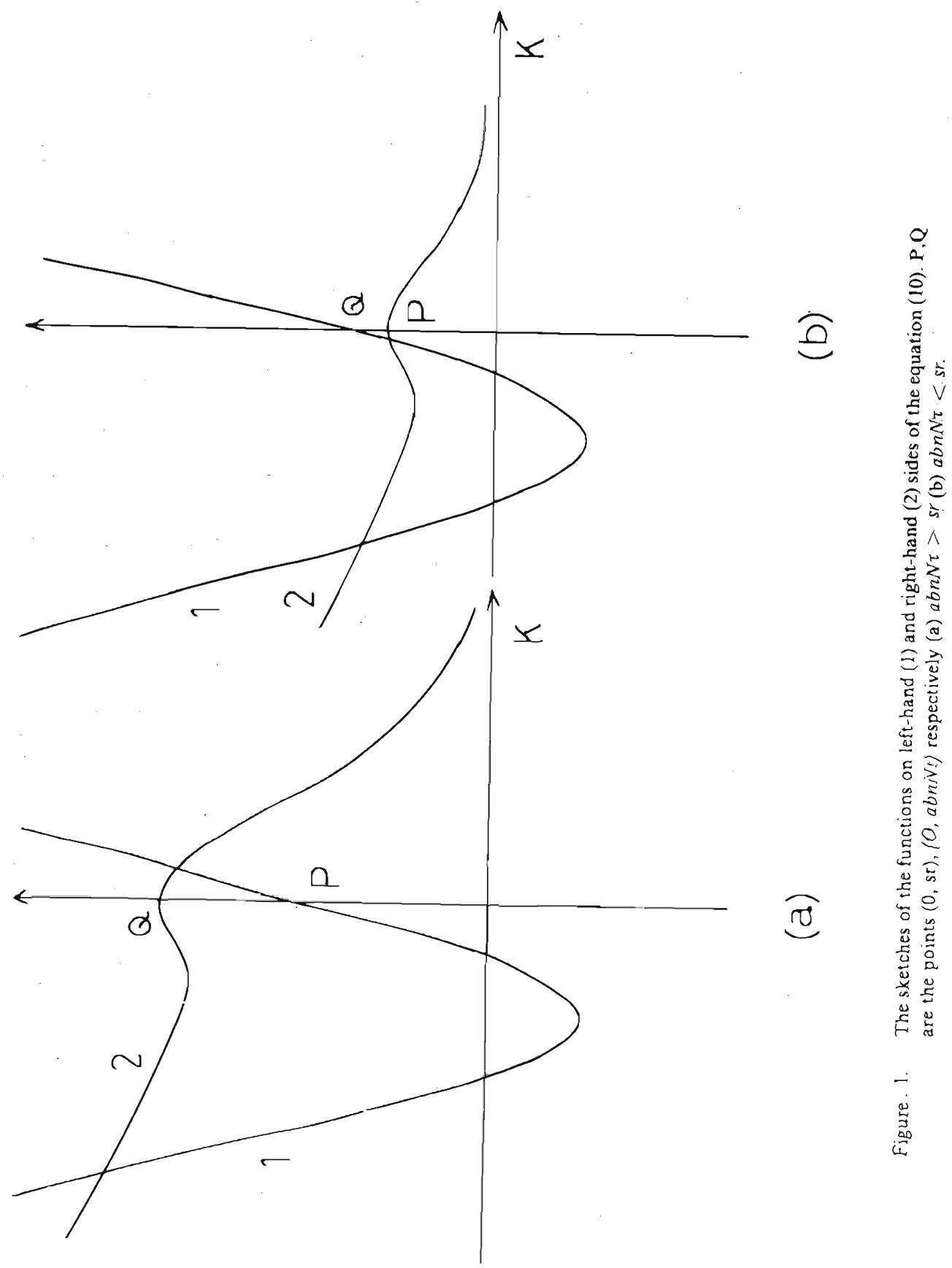

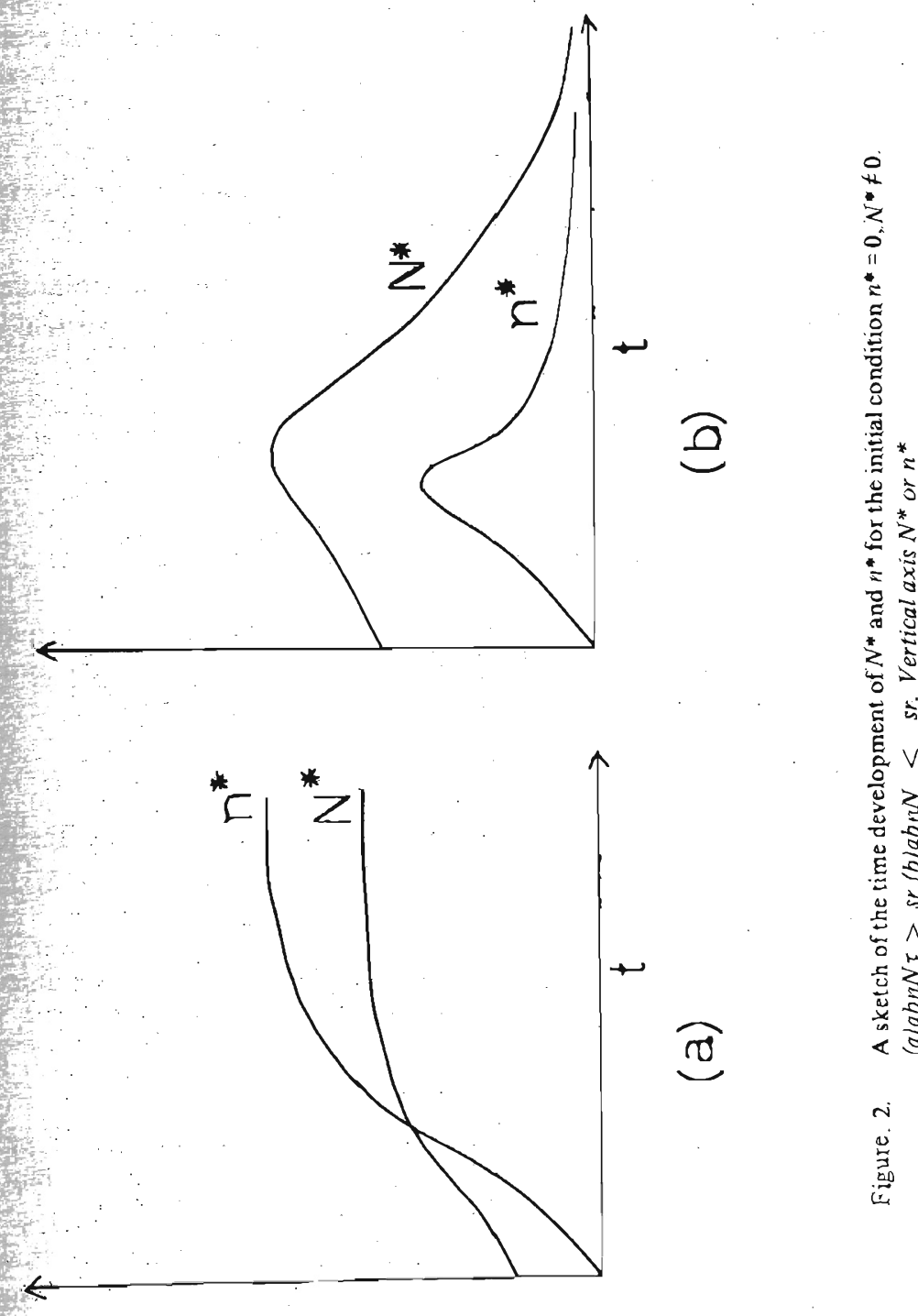
Where $A$ and $B$ are constants. When (11) is satisfied, the critical point $N^{*}=n^{*}=0$ is a saddle point and the system becomes unstable [11]. In the second case when both roots are negative, this critical point is an asymptotically stable node [11] (see Appendix). The consequences of these results are extremely important. When $n N$ the product of the population densities of nosguitoes and human beings exceeds the critical value $c=s r / a b r$, filariasis begins to develop explosively, but when this quantity is less thanc the disease decays, however, complete eradication is still difficult because of the asymptotic stability at the origin. From a knowledge of the behaviour of the system at the critical points it is possible to sketch the time development of $N^{*}$ and $n^{*}$ (Fig. 2).

The parameter $\tau$ is of the order of $9-12$ months and since we are interested in progress of the disease over years, the equation (10) can be approximated to a quadratic for $k \tau<1$ giving,

$$
\begin{aligned}
& k_{1}--(s+r) / 2-1 / 2 \sqrt{\left(s+r j^{2}-4(s r-a b n N \tau)\right.} \\
& k_{2}=-(s+r) / 2+1 / 2 \sqrt{(s+r)^{2}-4\left(s r-a b n N \tau_{1}\right)}
\end{aligned}
$$

Again as $-i_{m}^{\prime}$, where $t_{m} \sim 20 \cdot 30$ da!'s; is the mean life time of a mosquito, the term $e^{k}{ }_{1}$ in equation (13) decaying very fast can be neglected. Also $r^{-1}$, the mean time associated with the removal of infectives is larger than $s^{\mu}$, therefore.

$$
k_{2}=a b n N \tau / s-r
$$

Thus the solution near the critical point at the origin can be written in the form

$$
\left.N^{*} \bumpeq N_{0}^{*} e^{(a b n N \tau / s \cdots} r\right) t
$$

The above expression clearly shows, that the number of infectives exponentially increase or decrease according to as $\mathrm{Nn}>\mathrm{C}^{\prime}$ or $\mathrm{Nn} \cdot<\mathrm{c}$.

\section{Discussion}

The level of filarial infection prevalent today is sufficiently low and the man-parasitemosquito ecosystem probably does not develop very far from the critical point at the origin in the forward direction. In fact the data on incidence of filariasis in the southern coastal belt of Sri Lanka strongly favours the above hypothesis. In this region a continuous antifilarial campaign was active for the past 10 - 15 years. A significant portion of the population is being kept screened for filariasis (night blood test $^{4}$ ) and the positive cases are given drug treatment. Under these conditions, the constant $r$ in equation (1) rakes a value larger than its natural value resulting purely from immunological factors. Though not very successful mosquito control programmes are also carried out, this is roughly equivalent to increasing the value of the constant s. The plots of $h N^{*}$ 's $t$ for several urban and suburban areas in this 


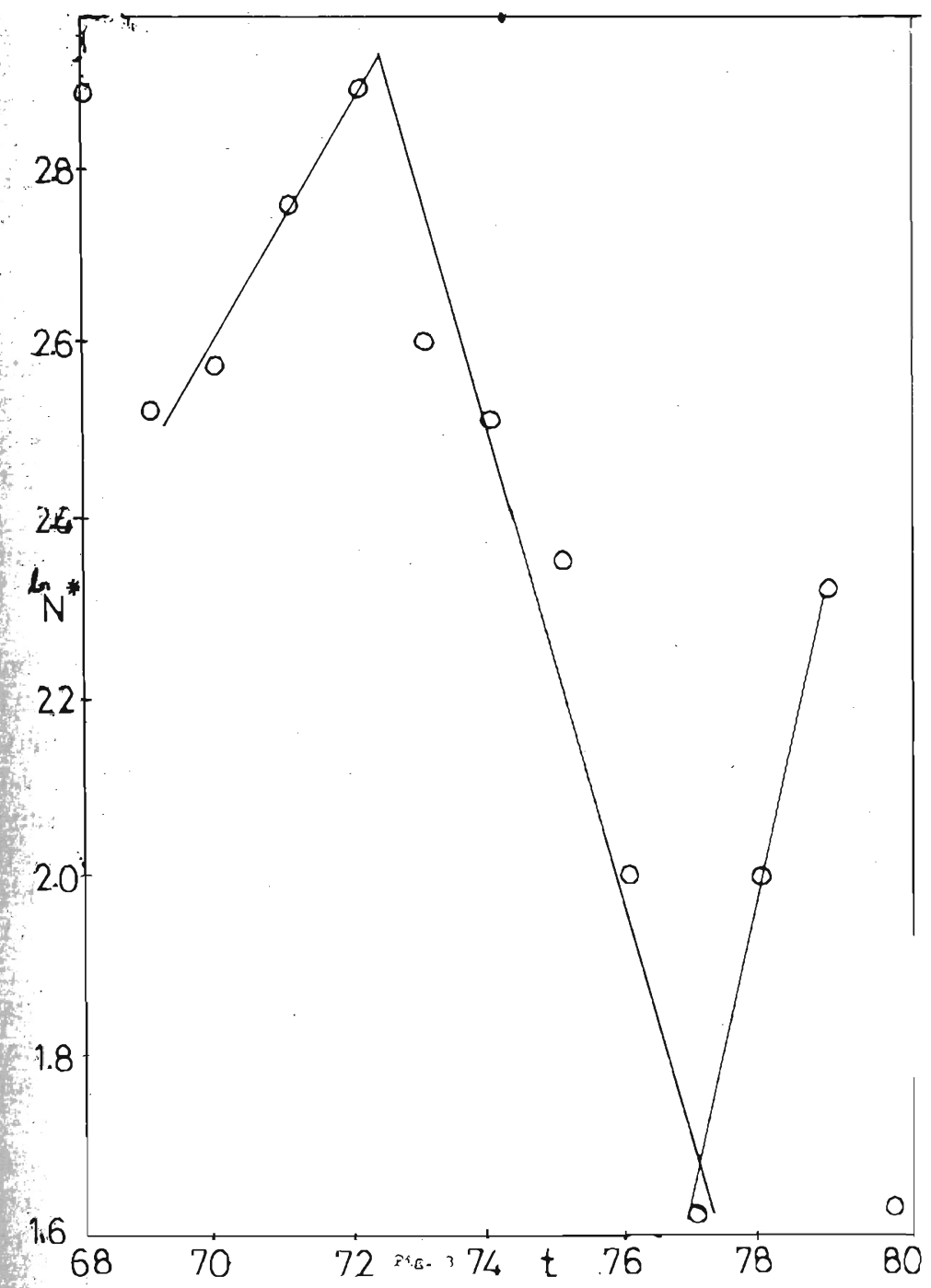

Figure 3. A plot of the natural logarithms of the number densities of filariasis cases detected per year from $1967-1980$ in a suburban region of area $36 \mathrm{~km}^{2}$ and average population 37.000 . The decay time of $N^{*}$ corresponding to the straight line portion in the middle is 5.1 years. 
region contain straight line portions with negative and positive slopes (Fig. 3). The lines with negative slopes corresponding to exponential decay of $N^{*}$ continues for longer intervals of time. Furthermore the numerical values of slopes are smaller for those areas with high population densities and more stagnation (e.g., larger value for $n N$ ). There is evidence that the equation (17) is satisfied, the general trend is towards an exponential decay of $N^{*}$ (Fig. 3). The sudden exponential increases are caused by abrupt changes in weather e.g., heavy rainfall. The data clearly illustrates the role playea by the quantity $n N$, that determines time development of the infection. Even in the exponential decreasing phase, a considerable level of infection occurs until a time.

$$
T=(r-a b n N \tau / s)-I
$$

The value for $T$ for the data presented in Fig. 3 is of the order 5 years. For a significant control of the disease, it is essential to reduce $T$ to a much ower value. The weather changes that drastically increase $n$ the population density of mosquitoes can be expected to occur in a period of 5 years and again the system becomes unstable with an exponential increase of the number of the infectives. It seems that the best way to eradicate the disease is to increase $r$ by intensifying the drug treatments and taking permanent measures to keep $n$ small by reducing stagnation.

If the quantity $n N$ is kept slightly above the critical value $c$, the infection could sustain itself indefinitely even if weather conditions adverse to the mosquitoes, lowers $n N$ below the critical value for short durations of time. The asymptotic stability of the origin erisures that complete eradication will not take place. Relatively large values of $r^{-1}$ and $\tau$ makes $c$ small, i.e, a low threshold value of $n N$ is sufficient for the survival of the host parasite system. Thus the pattern of the disease is different from that in malaria complete eradication becomes possible only if $n N$ is kept below this threshold for long intervals of time.

The model has other interesting features, that can be understood qualitatively. Even if the mosquito population is a periodic function of time (e.g. due to wet and dry seasons) there is a significant contribution to $d N^{*} / d t$ from the integral in the equation (l), when $\tau$ is large. The weather conditions sufficient to break the life cycle of the malarial parasite is not effective in eradicating filariasis. The study of this model when $n$ and $N$ are time varying functions could also be very relevant to the practical problem of eradicating the disease. However, in this case the equations (1) and (2) become nonautonomous [12] and the analytical discussion is extremely difficult.

\section{-Acknowledgements}

Author wishes to thank Prof. S. S. de Silva, Department of Zoology, Ruhuna University College, Matara. Sri Lanka for encouragement and the Matara Unit of the Sri Lanka Antifilaria Campaign for providing statistical data. 


\section{Appendix}

To study the nature of the critical point $N^{*}=N_{\infty}^{*} n^{*}=n_{\infty}^{*}$ we put $N^{*}=N_{\infty}^{*}+Y, n^{*}=n_{\infty}^{*} y^{\prime}$ in (1) and (2), where $Y, y$ are the deviations of $N^{*}, n^{*}$ from the steady values. After neglecting the second order terms in $y, Y$ we obtain,

$$
\begin{aligned}
& d Y / d t=b \int_{\mathrm{t}-\tau}^{\mathrm{t}}\left[p y(x)-n_{\infty}^{*} Y(x)\right] d x-r Y \\
& d y / d t=a q Y-u y
\end{aligned}
$$

Elimination of $Y$ between (A1) and (A2) yields,

$\left.d^{2} y / d t^{2}+(u+r) d y / d t+u r y=a b q\left(p-n n_{\infty}^{*} u / a q\right) \int_{t-\tau}^{\mathrm{t}} y(x) d x-b n_{\infty}^{*}(y / t)-y(t-\tau)\right\}(\mathrm{A} 3)$

Equation (A3) will have solutions of the form $y^{\prime}=($ constant $) \cdot e^{k t}$ provided

$k^{2}+(u+r) k+u r=(a q b / k)\left(p-n_{\infty}^{*} u / a q\right)\left(1-e^{-k t}\right)-b n_{\infty}^{*}\left(1-e^{-k t}\right)$

The above equation has two and only two roots, both real and negative (This can be easily shown, exactly in the same way as for the equation (10), by sketching curves similar to that in Fig: 1$)$ Thus the critical point $\left(n_{\infty}^{*}, N_{\infty}^{*}\right)$ is an asymptotically stable node.

\section{References}

1. BIRKHOFF, G. \& ROTA, G. C. (1978). Ordinary Differential Equations, 3rd. ed. John Willey, New York.

2. Chatter JEe, K. D. (1965) Parasitology, Sree Saraswathy Press, Calcutta.

3. CROFTON, H. D. (1971). A quantitative approach to parasitism, Parasitology 62: 179-193.

4. HAWKING, F. (1962). A Review of Progress in Chemotherapy and Control of Filariasis since 1955, Bull. WHO, $27: 551-568$.

5. JONES, A. W. (1967). Introduction to Parasitology', Addison Wesly, Massachussetts.

6. KESSEL, J. F. (1966). Fillariasis as a World Problem, Mosquito New's 26: 490499.

7. MAY, R. M. (1974). Stability and Complexity in Model Ecosystems, 2nd ed., Princeton U.P., Princeton. 

8. MAY, R. M. (1977). Thresholds and break points in ecosytems with a multipliciry of stable
Stares, Nature 269: 471 477 .

9. MACDONALD, G. (1957). The Epidemiology and Control of Malario, Oxford University Press
London.

10. SIMMONS, G. P. (1979). Differential Equations, Mc Graw-Hill, New York.

11. SMYTH, G. D. (1974). Introduction to Animal Parastology, Hodder and Stoughton, London. 12. WORLD HEALTH ORGANIZATION (1974). Expert committes on filariasis, Third report.
WHO Tech. Rep. Ser. No. 542 . 